\title{
Ueber einige Schwefeloxychloride *);
}

\section{von Friedrich Claussiser.}

(Mittheilung aue dom nouen chemischen Luboratoriom der Univernitut Tubingen.)

(Hionu Tafol I, Figur 1.)

H. Rose liefs mehrfach auf Chloride den Dampf des Schwefelsăureanhydrids einwirken und bekam so Doppelverbindungen, doren complicirte Zusammensetzung, besonders bei den Verbindungen des Schwefelchlcrids und Selenchlorids mit Schwefelsăureanhydrid, leicht $Z$ weifel in ihre Reinheit und Homogeneităt hervorrufen kopnten.

Wenn R os e wenig Schwefeisâureanhydrid zu auf $0^{\circ}$ abgekühltem und mit Chlor möglichst gesättigtem Chlorschwefel destillirte, so bekam er bei $1 \mathbf{1 5}^{\circ}$ siedendes Pyrosulfurylchlorid *t), beim Zusamnenbringen mit mehr Schwefelsăureanhydrid kam er zu einer festen krystallinischen Masse **) $\mathrm{SCl}_{8}, 30 \mathrm{SO}_{8}$, welche awar nicht dem Aussehen nach + ), wohl aber im Verhalten dem Schwefelsãureanhydrid ähnlich war und abgesehen von etwas „Mutterlauge", welche Rose am Boden des Gefăfses bemerkte, selbst nach 17 Jahren nicht verāndert erschien. Diese Angabe scheint im Widerspruch zu stehen zu den Beobachtungen von Millon und Michaelis, die beide auf ganz verschiedenen Wegen zu einem und demselben festen Schwefeloxychlorid $\mathrm{S}_{2} \mathrm{O}_{3} \mathrm{Cl}_{4}$ gelangten, das sich nach einiger

*) Inaugural-Diesertation, Tubingen 1878.

*4) Berrelius' Jabresbericht 19, 20; Pogg. Ann. 14, 291.

***) Jahresberioht fflr Chemie a. s. w. f. 1852, 850; Pogg. Ann. 8E, 810.

f) Nenerding hat $W$ o b e $($ Jabresber. Fir Chemio r. 8. w. f. 1876, 174) nachgewionen, dars die strahligen Schwofolehureanhydridtryotallo etwas wasserhaltig, dafs aber da reino Anhydrid in alpotor Whnlichen durchsichtigen Krystallen ascobiorst. 
Zeit unter Flüssigwerden zersetzte *). Millon * ) bekam seine „Chlorunterschwefelsäuro ${ }^{*}$ durch Einwirkenlassen feuchten Chlors anf Chlorschwefel; seine Angaben wurden spăter von Cariu s**) bestätigt. Michaolis t) stellte „Schwe(feloxytetrachlorid " dar in kleinen Mengen neben den Zersetzungsproducten $\mathrm{SOCl}_{2}, \mathrm{~S}_{2} \mathrm{O}_{6} \mathrm{Cl}_{2}, \mathrm{SO}_{2}$ und $\mathrm{Cl}$, indem er zu stark abgekühltem, mit Chlor möglichst gesättigtem Chlorschwefel eine concentrirte Lōsung von Schwefelsãureanhydrid in Schwefelsăure setzte, in jeder beliebigen Menge aber durch Einwirkenlassen von Chlor und Chlorschwefel auf abgekühltes Sulfurylhydroxylchlorid.

Nach diesen Untersuchungen erscheint es mir höchst wahrscheinlich, dafs eben die von $\mathrm{R}$ o s e erhaltene „Mutterlauge* aus den Zersetzungsproducten des in geringer Menge gebildeten $\mathrm{S}_{2} \mathrm{O}_{3} \mathrm{Cl}_{6}$ bestand, dafs aber alles Uebrige unverändertes Schwefelsäureanhydrid war.

Auf Selentetrachlorid wirkt nach Rosett) Schwefelsăureanhydrid in der Kälte wenig ein; in dor Wärme bekam er einen grüngelben Syrup, der bei stärkerem Frhilzen sich hellbraun färbte. Bei der Destillation desselben entwich zuorst das überschüssige Schwefelsäureanhydrid, dann ging als braunrother Dampf bei $187^{\circ}$ das, schwefelsaure Selenchlorid" über von der Zusummenselzung $2\left(\mathrm{SCl}_{8}, 5 \mathrm{SO}_{3}\right), 5\left(\mathrm{SeCl}_{4}, \mathrm{SeO}_{8}\right)$,

-) Nach Michaolis Bpaltang fn die Componenten $\mathrm{SO}_{2} \mathrm{Cl}_{8}$ und $\mathrm{SOCl}$ (Beriohte der deutsahen aheminchen Geselisohaft 1873, 1452).

*) Journal für praktisohe Chemie 18, 37; vputer Jabresber. für Chemio a. 8. F. f. 1850 , 275 ; Journal für praktisoho Chemio 80 , 267.

***) Diese Annalen 100, 295.

†) Carlsruher Habilitationssohrift. Giofsen 1873; dieve Annalen 1 10, 1.

††) Gmelin, Handb. d. Chem. 6. Auf. I, 2, 1t5; Pogg. Ann. 44, 815; Berrelias' Jabresber. IP, 204. 
welches nur durch mehrmalige Destillation gereinigt werden konnte.

Die Vermuthung, welche schon Berzelius in seinem Lehrbuch *) ausgesprochen hat, es sei dieser Körper einfach "schwefelsaures Selenchlorid" $\mathrm{SO}_{8}, \mathrm{SeCl}_{4}$ und durch "anhāngende Schwefelsāure " verunreinigt, lag jetzt nach den eingehenden Untersuchungen besonders von $\mathrm{Mi} \mathrm{ch}$ helis über das analoge Schwefeloxytetrachlorid vollends nahe und wurde mir nun zur Aufgabe, diese Selenverbindung rein darzustellen, ihre Eigenschaften und ihre Zersetzungen näher zu untersuchen, ihre Ar.alogie mit dem Schwefeloxytetrachlorid festzustellen und schliefslich im Schwefeloxytetrachlorid für ein Schwefelatom, wie hier Selen, so dem Schwefel und Selen mehr oder weniger verwandte Elemente and für die 4 Chloratome Brom eimzuführen.

Das zu meinen Versuchen erforderliche Sulfurylhydroxylchlorid stellte ich nach Michaelis **) durch Einwirkenlassen von Phosphorpentachlorid im status nascendi auf concentrirte Schwefelsãure dar. Die Fractionirung meines Rohproductes nahm ich, da bei langerem Sieden Zersetzung bemerkbar, in einem langhalsigen, von v. Miller ${ }^{* * *}$ ) vorgeschlagenen Kolben vor, dessen lange Destillationsröhre, weil die Kiorke sonst sehr stark leiden, weit in den Kühler hineinreichte. Die Eigenschaften des Sulfurylhydroxylchlorids fand ich übereinstimmend mit den von Michaelis u. A. angegebenen, mit Ausnahme des Siedepunktes, welchen ich mit verschiedenen feinen $G$ e if sler'schen Thermometern gemessen beim Barometerstand 726 MM. zu 150 bis $151^{\circ}$ fand (M i c h a eli s $158,4^{\circ}$ corrigirt; William son gegen $145^{\circ}$; Behrend $15^{\circ}$ ).

*) B. Auf. I, 210; Gmelin, Handb. d. Chem. 6. AnG. L, E, 416.

4.) Habilitationach rift (a. 0.).

mit) Diese Annalon 180, 184. 
Von den Thermometern befand sich das untere Drittel im Dampf; ihr Nullpunkt und Siedepunkt war controlirt. Dafs meine Substanz rein war, beweist die ausgeführte Analyse. (Die Substanz wurde wegen ihrer heftigen Zersetzung mit Wasser im Kugelröhrchen mit verdưnnter Salpetersăure und Silbernitrat in eine Rőhre eingeschmolzen.)

0,4620 Grm. Bubotanz gabon 0,5718 $\triangle \mathrm{gCl}$, oxteprechend $0,1412 \mathrm{Cr}$, and 0,9294 $\mathrm{BaSO}_{4}$ ontuprechond 0,1278 $\mathrm{B}$.

$\begin{array}{lcc} & \text { Bereohnet } & \text { Gefunden } \\ \text { C } & 80,47 & 30,57 \\ \text { B } & 27,46 & 27,62 .\end{array}$

Da ich vermuthete, dafs die Siedepunktsdifferenz zwischen meiner und $\mathbf{M}$ i ha $\mathrm{c}$ lis' Beobachtung vielleicht daher. rühre, daís die Messungen bei verschieden hohem Barometerstand ausgeführt warden, so machte ich Destillationsversuche unter vermindertem und erhöhtem Druck. Bei Anwendung eines Apparates mit Rūckflufskühler bemerkte ich aber, dafs schon bei gewỏhnlichem Luftdruck das Quecksilber im Thermometer langsam sank, weil theilweise Zersetzung in Schwefelsãure, Schwefligsăureanhydrid und Chlor eintrat, welch' letztere am Geruch deutlich erkennbar waren *). Ich machte deshalb meine Bestimmungen in einem einfachen Destillationsapparat mit einem v. Mille r'schen Fractionskolben und Abfluiskūhler. Die Beohachtungsergebnisse sind auf Tafel I graphisch dargestellt. Das Thermometer sinkt, wie man sieht, beim Fallen des Druckes um 20,6 MM., im Mittel nur um $1^{\circ}$.

Bevor ich Sulfurylhydroxylchlorid auf ein Chlorid des Selens einwirken liefs, stellte ich nach den Angaben von H i c h a e lis Schwefeloxytetrachlorid dar, dessen Eigenschaften ich den Angaben von $\mathbf{M}$ i chaelis ganz entsprechend fand.

") Willi a ms (nicht Williamson, wio falschlich in Gmolin's Handb. d. Chom. 6. Auf. I, D, 406 angegeben ist) rormuthoto Zersetsung in $\mathrm{SO}_{3}$ and CUR (Juurnal fur prattinaho Chemio 108, 124). 
Das bei Zimmertemperatur auf bewahrte Schwefeloxytetrachlorid war nach $2^{1 / 2}$ Monaten, das in der Kälte (bei wechselnden, im Allgemeinen aber niederen Temperaturen) aufbewahrte erst nach $3 \frac{1}{2}$, Monaten vollstāndig zerflossen *).

Im Lauf der Untersuchungen úber die dem Schwefeloxytetrachlorid analoge Selenverbindung hatte ich eine Anzahl Korper als Ausgangsmaterial nöthig, deren Darstellung und von mir năher untersuchte Eigenschaften ich hier angebe.

\section{Selen}

wurde nach Wöhler's * Vorschrift dargestellt aus 1000 Grm. von den Mansfelder Hüttenwerken bezogenem Selenflugstaub. Das durch eingeleitete schweflige Säure in der Siedhitze niedergeschlagene schwarze Selen wurde durch Destillation aus knieförmig gebogenen, am einen Ende zugeschmolzenen Verbrennungsröhren im Erlenmeyer'schen Verbrennungsofen gereinigt. Einige über das Selen geschichtete Glasscherben verhinderten ziemlich gut ein rasches Ueberschāumen. An Ausbeute bekam ich $240 \mathrm{Grm}$. reines Selen, also 24 pC. des Flugstaubs.

\section{Selentesrachlorid}

slellte ich am vortheilhaftesten dar durch Behandlang reinen Selens mit Chlor in circa $160 \mathrm{CC}$. langen Röhren. Sie waren am einen Ende offen zur Aufnahme eines dichtschliefsenden, mit Gasleitungsrohr versehenen einfach durchbohrten Korkes, am andern Ende zur offenen, mit einem Chlorcalciumrohr zur Abhaltung feuchter Luft verbundenen Spitze ausgezogen ; 20 CM. hinter dem durch Kork verschliefsbaren Ende war eine Verengung, welche einen stumpfen Winkel zwischen langem und

*) Entgegon dor Boobsehtung ron H. Boso (Pogg. Ann. 85, B10).

*) Gmelin, Handb. d. Chomie, 6. Aufl. I, D, 256. 
kurzem Rohrstack bildete. In das kurze Stack wurde in Portionen von 20 bis $30 \mathrm{Grm}$. das Selen gebracht, das Chlorid in das weite Stück hinaber sublimirt und dieses schliefslich abgeschınolzen. Das Abiỏsen der dicken Krusten des Sublimats hiefs sich leicht bewerkstelligen durch schwaches Klopfen an die Rőhre nach Erwärmung mit der Flamme.

Die Erscheinang, schon von Berzell us *) beobachtet, defs Selentetrachlorid beim Erhitzen einen gelben Dampf liefert, der sich um so dunkler färbt, je gröfser die Erhitzung, legte mir den Gedanken nahe, eine Dampfdichtebestimmung zu machen, um zu sehen, ob nicht, wia beim Phosphorpentachlorid von Deville und Cahours *) nachgewiesen war, auch hier Dissociation stattinde. Kelsulé ***) hat bekanntlich schon 1864 das Selentetrachlorid als "moleculare" Verbindung in die Reihe der in der Hitze dissociirenden Verbindungen gestellt zu Phosphorpentachlorid, Chlorammonium, Jodtrichlorid a. s. w. and dafür die Formel $\mathrm{SeCl}_{2}, \mathrm{Cl}_{2}$ geschrieben. Die Dampfdichtebestimmung nach Dumas ergab $d=3,922$ anstatt der berechneten Dichte $\mathrm{d}^{\star}=\mathbf{7}, \mathbf{6 3}$.

Somit ist die gefundene nahezu gleich der Hătfe der berechneten; es findet also Dissociation statl, wahrscheinlich nach der Gleichung :

$$
2 \mathrm{SeCl}_{4}=\mathrm{SO}_{8} \mathrm{Cl}_{8}+8 \mathrm{Cl}_{2} .
$$

Selenigsäureanhydrid, selenige Säure.

Reines Selenigsăureanhydrid erhielt ich, indem ich Selen in warmer Salpetersăure lōste, die Lösung zur Trockene verdampfte, den trockenen weifsen Rückstand in einem Kolben mit kurzem weitem Hals in einem Bad von Eisenfeile so lange

7) Gmolin, Handb. d. Chem. 6. Anf. I, D, 414.

*) Daselbet I, I, 244.

***) Compt. rend. 1864, 310. Stroit g॰gen Na oquet. 
erhitzte, bis sich keine Wasserdämpfe mehr an eizem kalten darũber gehaltenen Uhrglag condensirten und etwas Selenigsäureanhydrid in den Hals sublimirte.

Aus dem so erhaltenen Anhydrid läfst sich sehr leich selenige Süure herstellen; anstatt ersteres in $1 / 5$ seines $\mathrm{GB}_{0}$ wichts Wasser in der Siedhitze zu lösen *), was nicht sehr gut geht, löste ich das Anhydrid in überschüssigem heifsem Wasser und dampfte rasch über freier Flamme (wenn sich Spuren Selen ausschieden unter Zusatz eines Tropfens Salpetersäure) so lange ab, bis plötzlich das Entweichen von Wasserdämpfen aufhörte und die Flüssigkeit Syrupconsistenz hatte. Bei langsamem Erkalten schossen grofse Krystalle von seleniger Săure \&n zwischen ganz wenig Mutterlauge, von welcher dio Krystalle leicht durch Pressen zwischen Filtrirpapier gereinigt werden konnten.

Die kurze Notiz von W eb er**), wonacb selenige Săure an trockener huft verwittern soll, fand ich durch eigenen Versuch bestätigt; grofse Krystalle wurden über concentrirter Schwefelsăure nach einiger Zeit trüb undurchsichtig and trockenes Pulver von seleniger Șure zeigte bei mehrmaligem Wägen nach je 2 Stunden beträchtliche Gewichtsabnahme. Selenigsăureanhydrid und selenige Säure zieben on feuchtor Luft Wasser an, doch scheint letztere rascher zu zerfliefsen als ersteres.

Ueber das spec. Gewicht beider konnte ich keine Angaben finden, habe deshalb eine Bestimmung im Pyknometer vorgenommen. Als indifferente Flüssigkeit diente mir für beide Körper reines Benzol, in welohem sich Anhydrid und Săure weder lōsen noch zersetzen. (Unreines Benzol rom spec. Gewicht 0,8799 bei $17^{\circ}$ wird in Berührung mit beiden und

-) Gmolin, Fandb. d. Chom 4 6. Avf. I, 2 ; 264; Jahresbor. forr Chemio u. s. w \&. 1868, 158.

*) Dacelbst I, 8, 264; Jahresbex. fur Chomio u. s. w. f. $1868,168$. 
deren wăsseriger Lõsung in der Kalte langsam, in der Wârme rasch gelb und verursacht eine Rothfárbung der festen Săuren oder der wăsserigen Lösung in Folge sich ausscheidenden Selens.) Das reine Benzol hatte das spec. Gewicht 0,8839 bei $15,3^{\circ}$; thör $\mathrm{SeO}_{2}$ und $\mathrm{SeO}_{3} \mathrm{H}_{2}$ ergaben die Bestimmungen :

$$
\begin{aligned}
& \mathrm{B}_{\mathrm{O}}=8,9588 \text { bei } 15,8^{\circ} \text {, besogen anf Waner ron } \\
& \left.\mathrm{SeO}_{2} \mathrm{H}_{\mathbf{2}}=\mathbf{3 , 0 0 6 6}\right\} \text { gleicher Temperatur. }
\end{aligned}
$$

\section{Selenoxychlorid}

stellto ich dar durch Erhitzen von Selentetrachlorid und Selenigsåureanhydrid im Verhältnifs 1 Mol. auf 1 Mol., anfánglich nach W e ber's Angaben *) im knieförnig gebogenen zugeschmolzenen Rohr. Als aber in Folge davon, dafs gebildetes Selenorychlorid in den erhitzten Schenkel des Rohres zurückflors, in welchem die noch unzersetzten trockenen Substanzen lagen, ein Rohr zersprang, so versuchte ich durch einfaches Erhitzen der beiden Substanzen im richtigen Verhâltnifs im geraden Einscbmelzrohr die Zersetzung gefahrloser im Luftbad zu bewerkstelligen. In der That war nach mehrstündigem Erhitzen der Röhre auf $200^{\circ}$ Alles verfüssigt. Das immer in kleinem Ueberschưs absichtlich zugesetzte Selenigsäureanhydrid blieb beim Abdestilliren zurück. Das Selenoxychlorid ging alles constant beim Barometerstand 735 MM. bei 175 bis $176^{\circ}$ über (Weber gegen $220^{\circ}$; Michaelis $179,5^{\circ}$ ).

\section{Sulfurylchlorid.}

Dafs sich Sulfurylhydroxylchlorid bei längerem Sieden theilweise in $\mathrm{SO}_{4} \mathrm{H}_{2}, \mathrm{SO}_{8}$ und $\mathrm{Cl}_{2}$ zersetzt, habe ich bereits aus den oben angeführten Versuchen geschlossen. B e br e $\mathrm{d}^{* *}$ ) konnte selbst bei tagelangem Erhitzen keine Veränderung beobachten; Willia m son ${ }^{* * *}$ ) bekam $\mathrm{SO}_{2} \mathrm{Cl}_{2}$ und $\mathrm{SO}_{4} \mathrm{H}_{2}$. Wie

*) Gmolin, Hendb. d. Chom. 6. Auf. I, D, 114.

m) Journal für praktinche Chomio N. F. 16, 23.

av*) Daselbst $28,73$. 
man sieht, treten hier ganz verschiedene Resultate zu Tage und ist es vielleicht interessant, an dieser Stelle die Verhältnisse einmal näher zu beleuchten, unter welchen sich Chlor and schweflige Săure in freien oder im Entstehungszustande zu $\mathrm{SO}_{2} \mathrm{Cl}_{2}$ vereinigen, unter welchen nicht.

H. Schiff und Williamson *) geben an, durch Behandeln von $\mathrm{SO}_{3}$ oder Vitriolōl mit $\mathrm{PCl}_{5}$, Carius **) durch Erhitzen von $\mathrm{PbSO}_{4}$ mit $\mathrm{PCl}_{5}$ Sulfurylchlorid dargestellt zu haben. Michaelis **), Derselbe und Schifferdecker + ) und Geuther $f f$ ) bekamen neben Spuren von $\mathrm{SO}_{9} \mathrm{Cl}_{2}$ nur dessea Zersetzungsproducte $\mathrm{SO}_{\mathbf{y}}$ und $\mathrm{Cl}_{\mathbf{g}}$ durch Behandlung der bezüglichen Körper in offenen Gefäfsen.

Etwas anderes ist es, wenn die Zersetzung im zugeschmolzenen Rohr bewirkt wird. So bekam Gu st a v so $n+t+$ ), welcher Schwefelsãureanbydrid und Bortrichlorid zusammen im Binschmelzrohr erhitzte, Sulfurylchlorid nach der Gleichung :

$$
4 \mathrm{BO}_{8}+2 \mathrm{BCl}_{8}=8 \mathrm{SO}_{2} \mathrm{Cl}_{2}+\mathrm{B}_{2} \mathrm{O}_{3}, 8 \mathrm{O}_{2}
$$

und Behrend*), welcher Sulfurylhydroxylchlorid im Binschmelzrohr auf 170 bis $180^{\circ}$ erhitzte und eine Zersetzung nach der Gleichung :

$$
2 \mathrm{BO}, \mathrm{Cl}(\mathrm{OH})=\mathrm{BO}_{2} \mathrm{Cl}_{2}+\mathrm{BO}_{4} \mathrm{H}_{4}
$$

beobachtete, gründete darauf eine Darstellungsmethode des Sulfurylchlorids, welche bis zu 40 pC. der theoretischen Ausbeute lieferte.

Wie man aus diesen Resultaten ersieht, hăngt die Bildung von Sulfurylchlorid wesentlich davon ab, ob unter Druck und

-) Gmolin, Bandb. d. Chem. 6. Aufl. I, I, $40 \mathrm{D}$.

**) Daselbat 2, 405.

*w*) Jabresbor. flir Chomio u. s. w. f. 1870, 289 ; Jenaischo Zoitsohr. f. Mod. u. Nat B, 236.

f) Berichte der deutschon obomiechon Gosollwohaft 1879, 994.

t†) Daselbet 1872, 925.

t+†) Compt. rend. ro, 92.

-) Berichte der deutschen chominchen Gesellsoheft 1878, 1004. 
im gesohlossenen Gefäfs, oder bei Atmospherrendruck im offenen Cefäfs, in welchem schweflige Saure und Chlor Gelegenheit haben als Gase zu entweichen, operirt wird.

Dafs Caria 8 aufser durch Erhizen von braunem Chlorschwefel mit Schwefelsäureanhydrid in Einschmelzrohr auch durch Brwärmen von Phosphorpentachlorid mit schwefelsanren Salzen am Rückflufskühler anf 100 Sulfurylchlorid erhielt, ist immerhin möglich, da sich Chlor und schweflige Sāure, wio Regnault*) nachwies und Michaelis *) bestätigte, in Lichte vereinigen können zu Sulfurylchlorid. Unter welchen Bedingungen H. Schiff und Williams on gearbeitet haben, ist mir nicht bekannt.

Ein schönes Beispiel, wie die Bildung des Sulfurylehlorids vom Druek beeinflufst wird, haben wir an Schwefeloxytetrachlorid, das im offenen Gefars auf $57^{\circ}$ erhitzt in $\mathrm{S}_{2} \mathrm{O}_{3} \mathrm{Cl}_{4}$ nach der Gleichung :

$$
\left.4 \mathrm{~B}_{2} \mathrm{O}_{8} \mathrm{Cl}_{4}=\mathrm{B}_{2} \mathrm{O}_{8} \mathrm{Cl}_{2}+580 \mathrm{Bl}_{2}+2 \mathrm{Cl}_{2}+\mathrm{BO}_{2} \cdots *\right)
$$

zerfallt, während $\theta$ im geschlossenen Gefäfse nach einiger Zeit sich nach der Gleichung :

$$
\left.\mathrm{B}_{9} \mathrm{O}_{8} \mathrm{Cl}_{4}=\mathrm{sOCl}_{2}+\mathrm{8O}_{8} \mathrm{Cl}_{4} * *\right)
$$

zersetzt. Sulfurylchlorid entsteht also, um die verschiedenen Bildungsweisen kurz zusammenzufassen :

1) Bei Atmosphärendruck unter dem Einfluís des Sonnenlichts aus freiem Chlor und freier schwefliger Säure (Regnault, Michaelis).

2) Bei Atmosphärendruck aus schwefliger Saure und Chlor im Contact mit manchen Substanzen, so Aethylen (Regnault $f$ ), Kohle, krystallisirbarem Bisessig (Metsens) $+f$ ).

*) Borselin:' Jahreaher. 20, 64.

*) Jahresber. fir Chomic f. 1870, 288.

w4) Mi ob o elis, Habilitationswohrift (a. o.).

f) Berselias' Jahresber. 19, 198.

tf) Compt. rend. $8,92$. 
9) Bei hőherem Druck im geschlossenen frefáfs aus Verbindungen und Gemischen, welche im offenen Gefäfs bei höherer oder niederer Temperatur sich unter Abgabe von Chlor und schwefliger Saure zersetzen.

Für meine $\mathrm{Zwecke}$ bereitete ich mir Sulfurylchlorid nach der Methode von Melsens, indem ich in einer mit aufiteigendem Kühler versehenen tubulirten Retorte in krystallisirbaren Eisessig mehrere Stunden lang zu gleicher Zeit Chlor und schweflige Săure (letztere in kleinem Ueberschufs) einleitete, dann im Wasserbad abdestillirte, wieder einleitete und wieder abdestillirte. Das Destillat gab nach mehrmaliger Practionirung ein Product vom consianten Siedepunkt 72 bis $73^{\circ}$ (Re g n a ult $77^{\circ}$, Carius gegen $7^{\circ}$, Gustavs on 70 bis $71^{\circ}$, Michaolis und Schifferdecker 7009.

Bei den Destillationen konnte ich stets beobachten, dafs das Destillat anfänglich gelbgrân gefärbt erschien, sich aber nach einigen Tagen entfärbte (wabrscheinlich in Folge partieller Dissociation). Weiter fand ich, entgegen Ke $\mathrm{g} n$ a u $1 t^{\prime}$ 's Angaben, dafs Sulfurylchlorid wie Pyrosulfurylchlorid in Wasser von gewöhnlicher Temperatur untersinkt und sich nur langsam damit zersetzt in Salzsãure und Schwefelsãure.

\section{Sulfurylhydroxylchlorid und Selentetrachlorid.}

Die gesuchte Selendoppelverbindung durch Einleiten von Chlor in Sulfurylhydroxylchlorid, welches Selen suspendirt enthielt, darzustellen gelang nicht, da das Selen sich nur in geringer Menge mit tief dunkelgrüner Farbe in Sulfurylhydroxylchlorid löste Cohne Bildung von Schwelligsauureanbydrid in der Kälte), diese Fărbang versohwand beim Einleiten von Chlor; doch schien sich das am Boden liegende Selen nicht weiter zu verăndern.

Einen $z w e i t e n$ vergeblichen Versuch, zu Schwefelselenoxytetrachlorid 20 gelangen, machte ich nun, ausgehend von 
dem flüssigen Selenchlorür $\mathrm{Se}_{3} \mathrm{Cl}_{2}$. Zu diesem im Verhältnifs 1 Mol. $\mathrm{Se}_{2} \mathrm{Cl}_{2}$ auf 2 Mol. $\mathrm{SO}_{2} \mathrm{Cl}(\mathrm{OH})$ gebrachtes Sulfurylhydroxylchlorid wirkte anfangs heftig auf des Selenchloriur ein, unter Ausstofsen von Selentetrachloriddämpfen.

Beim Hindurchleiten von Chlor konnte neben Chlor und Salzsåure schweflige Săure am Geruch erkannt werden. Die Masse wurde mehr und mehr hell und dickflüssig, so dafs schliefslich kein Chlor mehr hindurchging.

Es blieb mir nun noch übrig, vom Selentetrachlorid ausgehend zu versuchen, ob sich daraus nicht die Selenverbindung rein gewinnen liefse. Ich brachte in einem langhalsigen schief gelegten Fractionskolben mit etwas aufwärts steigender Destillationsröhre zu $52 \mathrm{Grm}$. $\mathrm{SeCl}_{4}$ (1 Mol.) 27,5 Grm. SO $\mathrm{Cl}(\mathrm{OH})$ (1 Mol.) und schützte den Inhalt auf der einen Seite durch einen Kork, auf der andern darch ein vorgelegtes Chlorcalciumrohr gegen die atmosphärische Peuchtigkeit. Die Einwirkung ist keine sehr heftige, abzukühlen ist gar nicht nöthig, im Gegentheil das Genisch kühlt sich selbst ab. Momentan entweicht ziemlich viel Salzsãure, bald aber scheint es, als ob ein neu entstandener Körper das Selentetrachlorid umschliefse und vor weiterer Zersetzung schütze. Rrwärmt man im siedenden Wasserbad, so entweichen wieder Salzsăuredämpfe, allmälig hört aber nuch hier die Gasentwickelung auf, das uberschūssige unzarsetzte Selentetrachlorid setzt sich bald nach dem Schütteln in der Flüssigkeit wieder zu Boden. Jetzt erwärmte ich mit freier Flamme, es entwich neben Salzsāure Chłor und schweflige Sãure, die erhitzte Stelle bráunte sich. Nach 3 Stunden war ziemlich viel Selentetrachlorid an die obere Gefäfswand sublimirt. Die zurückgebliebene Masse war in der Kälte gelb, scheinbar trocken halbkrystallinisch, schmolz bei starkerem Erhitzen zur fast klaren rothen Flüssigkeit unter Abgabe von Strōmen Chlors und schwefliger Säure, welche in Wasser geleitet eine Menge Selen ausschieden. Beim Br- 
kalten erstarrte die Masse wieder gelblich halbkrystallinisch und sah zur Analyse nicht sehr einladend aus.

Ich hielt das Mischungsverhältnifs für ungeeignet und brachte deshalb diesmal zu $47 \mathrm{Grm}$. $\mathrm{SeCl}_{4}$ (1 Mol.) $49,8 \mathrm{Grm}$. $\mathrm{SO}_{2} \mathrm{Cl}(\mathrm{OH})(2 \mathrm{Mol}$ ). Schon in der Kălte war die Gasentwickelung lebhafter; nachdem diese aufgehört hatte erwărmte ich gelinde besonders das am Boden liegende Selentetrachlorid, welches sich langsam unter fortwährender Abgabe von Salzsăure auflöste. Allmälig mufste ich die Hitze steigern, denn je mehr Selenchlorid sich löste und je dickfússiger die Lösung wurde, um so schwieriger ging die Zersetzung des ersteren. In den entweichenden Gasen war jetzt auch wieder etwas Chlor und schweflige Sãure *) neben Salzsāure bemerkbar und es wurde namentlich bei dieser höheren Temperatur immer eine selenhaltige Verbindung mitgerissen, erkenntlich daran, dafs sich Selen am Ende der Gasleitungsröhre abschied. Die Flüssigkeit wurde besonders bei stărkerem Erwărmen dunkelgelb bis roth. Nach einiger Zeit hatte sich alles Selentetrachlorid gelöst, cine Sublimation von Selenchlorid hatte hier nicht mehr stattgefunden. Beim Erkalten verschwand die rothe Farbe und es erstarrte die gelbliche syrupartige Flüssigkeit vollstāndig zu einem mit Mutterlauge durchtrānkten, aus feinen

-) Dis Entstobung diewer boiden Geso, schon irther beobsohtot, könnte ihren Grund haben in Zersetzung der entatandonen Vorbindung nach der Gleichang $\mathrm{CiSO}_{8}-\mathrm{O}-\mathrm{BeCl}_{8}=\mathrm{SoOCl}_{8}+8 \mathrm{O}_{8}+\mathrm{Cl}_{\mathrm{z}}$, oder in Zersetzong oines Theils des Sulfurylbydroxyleblorids. Daff sich die Solondoppelrorbindung nioht nech obigor Gleichung zornotut haben kann, habe ich durch 2wrei directe Versuche bewiesen ; ich fand 1) dafe sich $\mathrm{BO}_{2} \mathrm{SeCl}_{4}$ fir wich orhitzt nicht sorsotzs (os findet bobohotens bei langerer golinder Emrurmang Dissociation in $\mathrm{BO}_{3}$ and $\mathrm{SeCl}_{4}$ statt), 2) dafs $\mathrm{Q}_{\theta} \mathrm{OCl}_{2}$ nioht noben $\mathrm{BO}_{8} \mathrm{Cl}(\mathrm{OH})$ exirtiron kann. Das einzig Mrgliche ist ensunehmen, dafo $\mathrm{BO}_{2} \mathrm{Cl}(\mathrm{OE})$ sich in eines soloh syrupertigen Fliseigteit sohon untor soinem Biedepunkt soreotst, anter $\mathrm{Abgabe}$ von $\mathrm{Cl}$ and $\mathrm{SO}_{\mathrm{g}}$ (bei geworhnliohem Luftdruck). 
meist radialfaserig gruppirten Nädelchen bestehenden Krystallkuchen. Ein Versuch, die Hasse durch mehrstündiges Hindurchleiten trockener Kohlensäure bei niederer und höherer Temperatur trocken zu erhalten, führte zu keinem Ziel. Burch Destillation das überschüssige Sulfurylhydroxylchlorid zu entfernen ging $z w a r$, allein der Rūckstand sowohl als die Selenverbindung hatte beim Erstarren nicht mehr das schöne krystallinische Gefüge, sondern bildete eine wachsäbnliche, compacte Masse. Die Reinigung zur Analyse wurde mit gutem Erfolg durch Trocknen auf weifsen sehr porösen Thonplatten unter einer Glocke über concentrirter Schwefelsâure vorgenommen. Die ursprünglich butterartig sich anfühlende Masse war nach 3 Tagen ein vollkommen trockener, aus weilsen seideglänzenden Nädelchen bestehender loser Krystallkucben *). Ein wiederholtes Trocknen auf diesen Platten war unnöthig und wäre auch wegen raschen Zerfliefsens des Körpers an feuchter Luft mit Schwierigkeiten verknüpft gewesen.

An die Luft gebracht zersetzt sich oie trockene Krystailmasse momentan, indem sie unter Abgabe von Salzsäure sich trübt und zu seleniger Saure und Schweîelsăure zersetzt; bald zerfliefst sie vollstündig zur klaren Flüssigkoit, welcht: sich in Wasser ruhig auflöst. Wirft man die Kryställchen direct in Wasser, so bemerkt man gelindes Zischen und rascht huflösung zur klaren Flüssigkeit. Bringt man wenig Wasser zu dis Selenverbindung, so ist die Einwirkung heftiger, so dafs momentan etwas Selentetrachlorid sublimirt, schliefslich löst sich auch hier Alles auf, meist ohne Selenausscheidung. Letztere bekounmt man dagegen immer, wenn Körper zugegen

*) Für moine opatteren Untersuchungen fand ich vortheilhsft, das $\mathrm{SO}_{8} \mathrm{BoCl}$ immer nur in Portionen, wolohe sich gat awischen einem Plattenpaar trocknen liefsen, darzastellen und ewar oinfach in langon weiten Roagensglesern mit angesetetem Destillirrohr. 
sind, welche sich mit Wasser noch rascher und unter gröfserer Wårmeerzeugung zersetzen, so Schwefelsäureanhydrid, Sulfurylhydroxylchlorid, Pyroschwefelsäure und sogar concentrirte Schwefelsáure, wenn letztere in grölserer Menge vorhanden; heifses Wasser zersetzt diese Verbindungen, wie Mich a eli s *) am Schwefeloxytetrachlorid gezeigt, in anderer Weise als kaltes. Rose **), dessen „schwefelsaures Selenchlorid“ mit Wasser zersetzt meist Selen ausschied, hatte offenbar den durch Schwefelsãureanhydrid verunreinigten Körper.

Da beim Zusammenbringen der Substane mit Wasser zu heftige Reaction stattindet, so liers ich zum Zweck der Analyse den Körper einfach zerfliefsen. Die Lösung wurde sorgfaltig in ein Becherglas gespült und mit den Waschwãssern verdünnt; sie roch selbst beim Erwärmen nicht nach Chlor oder schwefliger Säure und enthielt nur Schwefelsäure, selenige Sãure und Salzsãure. Ich versetzle zuerst die heifse sehr verdünnte Lòsung mit etwas Salpetersăure und Baryumnitrat, um die Schwefelsäure als Baryumsulfat, das Filtrat mit Silbernitrat, um die Salzsãure als Chlorsilber zu fâllen.

Das orhaltene Baryumsulfat wurde nach dem Glūhen mit heifser Salzsāure ausgezogen. Das Selen wurde in einer neuen Portion durch Ausfällen aus kochender Lösung mittelst schwefliger Sãure bestimmt.

1,3343 Grm. Substanz lieferten 1,0521 BagO, und 2,4907 AgCl.

1,0446 Grm. Bubstanz gaben 0,2779 8e.

Diese Worthe enteprechen nahezn der Formel $\mathrm{8O}_{8} \mathrm{BoCl}_{4}$ :

$\begin{array}{lcc} & \text { Berechnet } & \text { Gefunden } \\ \text { s } & 10,63 & 10,82 \\ \text { Cl } & 47,17 & 46,15 \\ \text { Se } & 26,24 & 26,60 .\end{array}$

Somit habe ich wirklich hier den dem Schwefeloxytetrachlorid

*) Habilitationsaclirift (o. 0.).

**) Pogg. Ann. 44, 304. 
analog zusammengesetzten Körper Schwefolselenoxytetrachlorid

$$
\mathrm{ClSO}_{2}-\mathrm{O}-\mathrm{SeCl}_{2}
$$

und das bei der Darstellung desselben im Uebersohufs zugesetzte Sulfurylhydroxylchlorid dient lediglich als Lossungsmittel *), ist aber nōthig, damit vollkommene Vereinigung des Selentetrachlorids mit Sulfurylhydroxylchlorid stattindet.

Obgleich das Schwefelselenoxytetrachlorid zweifelsohne dem Schwefeloxytetrachlorid analog zusammengesetzt ist und somit als Selentetrachlorid betrachtet werden kann, in welchem ein Atom Chlor durch das Radical $\mathrm{ClSO}_{2}-\mathrm{O}_{-}$, oder als Sulfurylchlorid, in welchem ein Atom Chlor durch das Radical $\mathrm{Cl}_{9} \mathrm{Se}-\mathrm{O}$ substituirt ist, so zeigen sich doch grofse Unterschiede in ihrem Verhalten; so schon bei ihrer Bildung : $\mathrm{S}_{2} \mathrm{O}_{3} \mathrm{Cl}_{4}$ entsteht nur in der Kălte und zersetzt sich langsam bei Zimmerlemperatur, rasch beim Erhitzen bis zum Schmelzpunit (570) in die Producte $\mathrm{S}_{2} \mathrm{O}_{3} \mathrm{Cl}_{2}, \mathrm{SOCl}_{2}, \mathrm{Cl}_{2}$ und $\mathrm{SO}_{2}$ oder $\mathrm{SO}_{2} \mathrm{Cl}_{2}$ und $\mathrm{SOCl}_{2}$.

$\mathrm{SSeO}_{3} \mathrm{Cl}_{4}$ hat zu seiner Bildung Wärme nöthig und zersetzt sich weder von selbst bei Zimmertemperatur oder im Sonnenlicht (selbst nicht nach Monaten), noch bei Anwendung von Wärme und nur sehr schwer bei Einwirkung vieler anderer Substanzen, welche auf das $\mathrm{S}_{2} \mathrm{O}_{3} \mathrm{Cl}_{4}$ heftig zerselzend einwirken. Den Schmelzpunkt des reinen $\mathrm{SSeO}_{3} \mathrm{Cl}_{4}$, welches ich zu diesen Bestimmungen in Röhrchen einschmelzen mufste, fand ich bei 1650. Der Körper schmilzt zur rothen, je vach der Temperatur mehr oder minder dunkeln klaren Flüssigkeit und destillirt bei $183^{\circ}$ (Rose $187^{\circ}$ ) ohne Abgabe von Chlor und schwefliger Saure als braunrother Dampf, der sich schon oben in der Destillirröhre verdichtet zum Syrup, welcher, wenn er nicht

-) 1 Mol. 80,ClH sa 1 Mol. BeCl gobracht geben sohon bei geringer Erwarmung oine klare Losung, ans wolcher in dor Kalto Nichte aukkyutallisirt. 
durch erhebliche Mengen Schwefelsãureanhydrid verunreinigt ist *), sogleich zur gelblichweifsen wachsăhnlichen Masse von undeutlich krystallinischer Structur erstarrt. Die braune, dem Dampf des Selentetrachlorids so âhnliche Farbe des gasförmigen $\mathrm{SSeO}_{3} \mathrm{Cl}_{4}$ liefs mich vermuthen, es möchte das letztere hierbei dissociirt sein in $\mathrm{SO}_{3}$ einerseits und $\mathrm{SeCl}_{4}$ resp. $\mathrm{Se}_{2} \mathrm{Cl}_{2}$ und $\mathrm{Cl}$ andererseits. Bine wie beim Selentetrachlorid ausgeführte Dum as'sche Dampfdichtebestivomung lieferte mir bei $209^{\circ}$ die Zahl :

$$
\begin{aligned}
& d=8,362 \text { anstatt der bereohneton : } \\
& d^{\prime}=10,426 ;
\end{aligned}
$$

somit ist die gefundene Dampfdichte gleich ungefäir $1 / 3$ der berechneten und fand also, wie ich vermuthet hatte, Dissociation statt, wahrscheinlich nach der Gleichung :

$$
2 \mathrm{BO}_{3} \mathrm{BeCl}_{4}=2 \mathrm{SO}_{3}+\mathrm{Se}_{8} \mathrm{Cl}_{8}+8 \mathrm{Cl}_{20}
$$

welche Zersetzungsproducte sich in der Kälte wieder 20 $\mathrm{SSeO}_{8} \mathrm{Cl}_{4}$ vereinigen; denn es schlägt sich der in der Hitze braune Dampf beim Erkalten als fester, weifser, schmelzbarer Körper nieder.

Auch gegen Wasser verhalten sich $\mathrm{SSeO}_{3} \mathrm{Cl}_{4}$ und $\mathrm{S}_{2} \mathrm{O}_{3} \mathrm{Cl}_{4}$ verschieden; beide zersetzen sich rach damit, während aber letzteres **) mit wenig Wasser neben schwefliger Săure und Salzsäure Thionylchlorid und Pyrosulfurylchlorid resp. Sulfurglhydroxylchlorid giebt, zersetzt sich die Selenverbindung gerado als ware sie ein Gemisch aus $\mathrm{SO}_{8}$ und $\mathrm{SeCl}_{4}$ ), es wird das $\mathrm{SO}_{8} 2 \mathrm{zu} \mathrm{SO}_{4} \mathrm{H}_{2}$ und unter Abgabe von Salzsãure das $\mathrm{SeCl}_{4}$ zu $\mathrm{SeOCl}_{2}$ und $\mathrm{SeO}_{2}$ und schliefslich $\mathrm{SeO}_{3} \mathrm{H}_{2}$. Eine Zersetzung analog der des $\mathrm{S}_{2} \mathrm{O}_{3} \mathrm{Cl}_{4}$ in $\mathrm{SO}_{3} \mathrm{ClH}$ und $\mathrm{SeOCl}_{2}$ ist nicht mõglich, da diese (siehe unten) nicht neben einander existiren kōnnen.

*) $\mathrm{SO}_{8}$ drutckt aufserdem den Biedepankt otwan berab.

- Michaelir, diese Annalen 120, 22.

***) Diese Bpaltung int unter Umstanden siohtbar (sioho ohen). 
Concentrirte Schwefelsäure wirkt auf $\mathrm{S}_{9} \mathrm{O}_{8} \mathrm{Cl}$, heftig ein, unter Bildung von schwefliger Sãure, Salzsãure und Sulfurylhydroxylchlorid nach der Gleichung :

$$
\mathrm{B}_{2} \mathrm{O}_{8} \mathrm{Cl}_{4}+2 \mathrm{gO}_{4} \mathrm{H}_{8}=8 \mathrm{SO}_{2} \mathrm{Cl}(\mathrm{OH})+\mathrm{SO}_{8}+\mathrm{ClH} \text { (Michaelis). }
$$

$\mathrm{SSeO}_{3} \mathrm{Cl}_{6}$ wird von reiner Schwefelsäure $\mathrm{SO}_{4} \mathrm{H}_{2}$ (dargestellt durch mehrmaliges Umkrystallisiren der unter $\infty^{\circ}$ aus schwach rauchendem Vitriolöl erhaltenen Krystalle; Schmelzpunkt gegen $+10^{\circ}$ ) in der Kälte gar nicht, bein Erwărmen kaum zersetzt. Die Selenverbindung löst sich dabei in einem ziemlichen Ueberschufs an Schwefelsäure, ohne in der Kälte wieder auszukrystallisiren, unter Abgabe von etwas Salzsüure *), erhitzt man höher, so destillirt das $\mathrm{SSeO}_{8} \mathrm{Cl}_{4}$ unverändert bei $183^{\circ}$; von gebildetem Sulfurythydroxylchlorid, Selenoxychlorid, schwefliger Saure oder Chlor war Nichts zu bemerken, es zersetzt sich somit die Selenverbindung mit Schwefelsãure nur in ganz geringer Menge und zwar nach den Gleichungen :

$$
\begin{aligned}
& \mathrm{SBeO}_{2} \mathrm{Cl}_{4}+3 \mathrm{SO}_{4} \mathrm{H}_{2}=2 \mathrm{~B}_{2} \mathrm{O}_{4} \mathrm{H}_{2}+\mathrm{SeOCL}_{4}+2 \mathrm{ClH} ; \\
& \mathrm{SeOCl}_{2}+2 \mathrm{SO}_{4} \mathrm{H}_{2}=\mathrm{B}_{2} \mathrm{O}_{7} \mathrm{H}_{2}+\mathrm{BeO}_{2}+2 \mathrm{CHH} .
\end{aligned}
$$

Selenigsäureanhydrid konnte in der rückständigen Scbwefelsăure nachgewiesen werden.

Alle diese Unterschiede im Verhalten der beiden Körper $\mathrm{S}_{2} \mathrm{O}_{3} \mathrm{Cl}_{4}$ und $\mathrm{SSeO}_{8} \mathrm{Cl}_{4}$ sind weitere Baweise für den von Michaelis**) aufgestellten Satz :

„Die Affinität des Selens zu Chlor ist bei weitem gröfser als die zu Sauerstoff, die Affinitäten des Stickstoffs und Sauer-

*) Iah konnto immer, auch apłter, bemerkeu, dafs, sobald Gase wio 7. B. hier Salegäure aus einer Lösung des $\mathrm{SSeO}_{8} \mathrm{Cl}_{4}$, welche nicht viel $\mathrm{SO}_{3}$ enthielt, entwichen, diese Selentetracblorid mit sich risen, dag sich sls leichter Anflug an die oberen Gefarkwando legto. Dio Verbindung scheint also anch hier in geringer Menge zu dissociiren in $\mathrm{SO}_{3}$, wolches in der Flübsigkeit $\left(\mathrm{SO}_{4} \mathrm{H}_{2}\right.$ u. s. w.) surückbleibt, und $\mathrm{SeCl}_{4}$, welches im Gasstrom sublimirt.

**) Jenaigche Zeitschrift f. Med. und Nat. B, 86. 
stoffs zu Selen sind kleiner, die des Chlors dagegen gröfser als die Affinitäten dieser Körper zu Schwefel."

Zu gleichem Resultat über die Verwandtschaftsverhältnisse des Schwefels und Selens gegen Sauerstoff und Chlor gelangte ich, als ich das Verhalten des Schwefelchlorürs gegen Selenigsäureanhydrid untersuchte. $\mathrm{SeO}_{\mathbf{q}}$ löst sich in der Kälte vollständig in $\mathrm{S}_{\mathbf{\alpha}} \mathrm{Cl}_{9}$, unter Abgabe von $\mathrm{SO}_{\S}$ und Zersetzung nach der Gleichung :

$$
2 \mathrm{SeO}_{3}+2 \mathrm{~B}_{8} \mathrm{Cl}_{2}=2 \mathrm{SeOCl}_{2}+8 \mathrm{O}_{2}+\mathrm{S}_{3} \text {. }
$$

Der Schwefel bleibt in überschüssigem $\mathrm{S}_{\mathbf{q}} \mathrm{Cl}_{2}$ gelōst. Die Zersetzung geht aber noch weiter; die durch $\mathrm{S}_{8} \mathrm{Cl}_{8}$ ursprünglich gelbroth gefärbte Flüssigkeit wurde bald in Folge von gebildetem $\mathrm{Se}_{2} \mathrm{Cl}_{2}$ dunkelbraunroth und nach mehreren Stunden hatte sich sogar $\mathrm{SeCl}_{4}$ ausgezchieden. Ein directer Versuch, bei welchein ich $\mathrm{S}_{2} \mathrm{Cl}_{2}$ auf $\mathrm{SeOCl}_{2}$ einwirken liefs, bestätigte mir die Zersetzung nach der Gleichung :

$$
2 \mathrm{~S}_{6} \mathrm{OCl}_{2}+\mathrm{S}_{2} \mathrm{Cl}_{2}=\mathrm{Se}_{2} \mathrm{Cl}_{2}+2 \mathrm{SOCi}_{2} \text {; }
$$

$\mathrm{SOCl}_{2}$ und $\mathrm{SeOCl}_{2}$ wirken aber, wie $\mathrm{Michaelis} \mathrm{*)} \mathrm{fand} \mathrm{und}$ wie ich nach wiederholtem Versuch bestătigen kann, nach folgender Gleichung auf einander ein :

$$
\mathrm{SOCl}_{x}+\mathrm{SeOCl}_{2}=\mathrm{gO}_{8}+\mathrm{SeCl}_{4}
$$

Sulfurylchlorid und Solonoxychlorid.

Diese Resultate legten mir den Gedanken nahe, zur Darstellung des Schwefelselenoxytetrachlorids einmal den umgekehrten Weg einzuschlagen, d. h. anstatt im Selentetrachlorid ein Chloratom durch ein Sauerstoffaquivalent des Schwefelsäureanhydrids zu substituiren, im Selenoxychlorid ein Sauerstoffäquivalent gegen ein Chloratom des Sulfurylchlorids umzutauschen.

Selenoxychlorid und Sulfurylchlorid ungefähr im Verhäli-

*) Gmolin, Handb. d. Chem. 6. Anf. I, D, 415. 
nifs 1 Mol. auf 1 Mol. zusammengebracht wirken anfangs merkwürdiger Weise gar nicht auf einander ein. Die beiden Substanzen wizchten sich zur grünlichgelben Flüssigkeit, welche in ein Robr eingeschmolzen nach einigen Tagen kleine glänzende, . stark lichtbrechende, würfelähnliche *), unter dem Mikroscop wie kurze rhombische Säulchen (mit Rhombenpyramide, makrodiagonalem Flächenpaar und basischer Fläche) aussehende Krystalle uusschied, die sich allmälig noch ein wenig vermehrten und vergrofserten und sich, als ich sie an der Rohrwand von anhängender Flüssigkeit mit der Flamme trocknete und nun störker orhitzte, ohne zu schmelzen, unter Zusammensintern und Gelbfärbung verflūchtigten, also nicht $\mathrm{SSeO}_{8} \mathrm{Cl}_{4}$, sondern einfach krystalligirtes Selentetrachlorid waren **). Nach mehrtăgiger Binwirkung des Sonnenlichts konnte keine weitere Veränderung beobachtet werden; dagegen zeigte sich schon nach mehrstündigem Erhitzen der Röhre anf 170 bis $180^{\circ}$ nahezu Alles in wenig überschūssiger Flūssigkeit erstart zu schönen săulenförmigen Kryställchen, welche nun beim Brhitzen zur rothgelben Flūssigkeit schmolzen and sich nach niherer Untersuchung als Schwefelselenoxytetrachlorid herausstellten. In der Kälte scheint alsn der Procefs zu verlaufen nach der Gleichung :

$$
\mathrm{sO}_{2} \mathrm{Cl}_{2}+\mathrm{seOCl}_{2}=\mathrm{sO}_{3}+\mathrm{BeCl}_{4} \text {. }
$$

Das $\mathrm{SO}_{8}$ setzt sich wahrscheinlich im status nascendi mit $\mathrm{SO}_{8} \mathrm{Cl}_{8}$ um zu $\mathrm{S}_{2} \mathrm{O}_{6} \mathrm{Cl}_{2}$, welches zwar nicht in der Kälte, wohl aber, wie ich später (siebe Seite 287) nachgewiesen habe, in der

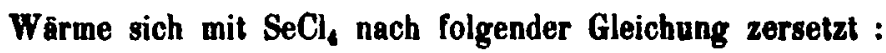

-) Michaolis bekam ans hoifsem Phouphoroxychlorid $\mathrm{BoCl}_{4}$ in glknzenden Wtirfeln krystallinirt (Gmelin, Handb. d. Chom. 6. Anf. I, \$, 116).

**) Bei der Danstellung des $\mathrm{BeOCl}$, wurdo immer (wiobe oben) otwas mehr als dio aquiralento Monge $\mathrm{BoO}_{2}$ angervendet; os konnte somit jenes nicht wohl tberschügsiges $\mathrm{S} \times \mathrm{Cl}_{4}$ gelöat enthalten. 


$$
\begin{aligned}
& \mathrm{B}_{8} \mathrm{O}_{6} \mathrm{Cl}_{4}+\mathrm{SeCl}_{4}=\mathrm{SO}_{2}+\mathrm{Cl}_{4}+\mathrm{SO}_{2} \mathrm{BeCl}_{6} ; \\
& \text { oder suob (wio hior) }=\mathrm{BO}_{2} \mathrm{Ch}+\mathrm{BO}_{8} \mathrm{BeCl}_{4} \text {. }
\end{aligned}
$$

Würde also das Flüssigkeitsgemisch sogleich auf 170 bis $180^{\circ}$ erhitzt, so würde der Procefs glatt nach der Gleichung verlaufen :

$$
\mathrm{BO}_{2} \mathrm{Cl}_{4}+\mathrm{SeOCl}_{3}=\mathrm{BO}_{8} \mathrm{SOCl}_{4}
$$

\section{Pyroschwefelsäure und Selentetrachlorid.}

Nachdem concentrirte Schwefelsäure kaum auf $\mathrm{SSeO}_{3} \mathrm{Cl}_{4}$ eingewirkt hatte, schien es wahrscheinlich, dafs man, das Sulfurylhydroxylchlorid umgehend, die Selenverbindung auch erhalten kônne durch Einwirkenlassen von Selentetrachlorid anf Pyroschwefelsăure $\mathrm{S}_{2} \mathrm{O}_{7} \mathrm{H}_{2}$. Letztere stellte ich dar durch Einleiten von Schwefelsäureanhydriddāmpfen in reines rauchendes Vitriolöl, bis das Product nahezu den Schmelzpunkt $+35^{\circ}$ (M a rign a c *) hatte. Pyroschwefelsăure (auf $40^{\circ}$ erwärmb) und Selentetrachlorid im Verhättnifs $1 \mathrm{Mol}$. auf 1 Mol. wirken nicht auf einander ein, erst beim Erwärmen löst sich alles Selentetrachlorid, wobei im Anfang keine Gasentwickelung, zuletzt schwache Salzsăureabgabe zu bemerken isi, zur klaren dicklichen Flüssigkeit, aus welcher beim Erkalten nicht so rasch **) wie aus einer Auflōsung in Sulfurylhydroxylchlorid, schliefslich aber doch in ganz derselben Form, das $\mathrm{SSeO}_{6} \mathrm{Cl}_{4}$ (vom Siedepunkt $183^{\circ}$ ) auskrystallisirt. Der Procefs ist also in grofsen Ganzen folgender :

$$
\mathrm{S}_{2} \mathrm{O}_{7} \mathrm{H}_{2}+\mathrm{BeCl}_{4}=\mathrm{BO}_{3} \mathrm{BeCl}_{4}+\mathrm{SO}_{4} \mathrm{H}_{2} \text {. }
$$

Das Auftrten geringer Mengen Salzsãure läst sich aus der Einwirkung der entstandenen Schwefelsãure auf Selentetrachlorid (siehe unten), oder aus der Einwirkung von Schwefel-

*) Gmel i n, Handb. d. Chem. 6. Anf. I, D, 195.

*) Ein Gohalt des Lösungsmittels an $\mathrm{SO}_{8}$ verhindert oder verzögert wenigatons die Krystallisatiou des $\mathrm{SSeO}_{3} \mathrm{Cl}_{6}$. 
säure auf die schon gebildete Seìnverbindung (siehe oben) erklären.

Es ist diese Darstellungsweise eine sehr einfache, man hat nicht die zeitraubende und mit gröfseren Kosten verbundene Bereitung des Sulfurylhydroxylchlorids nōthig und kommt zu demselben reinen Product, vorausgesetzt, dafs man reine Pyroschwefelsãure hat, deren Herstellung nicht schwicrig und defs die richtigen Verhältnisse genommen werden.

Die Anwendung einer Pyroschwefelsăure, welche überschūssige Schwefelsãure enthält, ist immer vortheilhafter als die einer solchen, welche überschüssiges Schwefelsturcanhydrid enthålt.

\section{Schwefelsäure und Selentetrachlorid.}

Schwefelsăure $\mathrm{SO}_{4} \mathrm{H}_{2}$ allein ohne einen Gehalt an Anhydrid führt sogar, wenn man sie auf Selentetrachlorid einwirken läfst, zum Schwefelselenoxytetrachlorid. Bine Mischung von Schwefelsäure und Selentetrachlorid im Verhältnifs 2 Mol. auf 1 Mol. schäumt beim Erwärmen zu sehr (in der Kälte bemerkt man keine Zersetzung), ich erwărmte daher ca. gleiche Volumina der beiden Körper zusammen; das Selentetrachlorid lōste sich unter Abgabe von viel Salzsâure rollständig, wobei wieder die schon oben besprochene Dissociationserscheinung zu bemerken war, zur klaren gelben Flüssigkeit, welche beim $\mathrm{Er}$ kalten nicht erstarrte. Erhitzt man die Lösung stärker und giebt dem gebildeten Schwefelsâureanhydrid Gelegenheil zu entweichen, so kann man nun bei $183^{\circ}$ das $\mathrm{SSeO}_{3} \mathrm{Cl}_{4}$, freilich noch mit elwas $\mathrm{SO}_{8}$ verunreinigt, abfractioniren, es erstarrt dann in der Destillirröhre. Fractionirt man rasch ab, so geht mehr $\mathrm{SO}_{3}$ mit dem $\mathrm{SSeO}_{3} \mathrm{Cl}_{4}$ bei circa $180^{\circ}$ über und gesteht dann die Masse zum gelblichwoifsen, nicht krystallisirenden Syrup fje nach den Verhältnissen auch zur halbbarlen, horn- 
artigen Masse). Dic Reaction verlāuft hauptsăchlich nach folgender Gleichung :

$$
3 \mathrm{SoCl}_{4}+2 \mathrm{SO}_{4} \mathrm{H}_{3}=\mathrm{SeO}_{3}+2 \mathrm{SO}_{3} \mathrm{SeCl}_{4}+4 \mathrm{ClH}
$$

ein Theil des Selentetrachlorids wirkt aber auch nur anhydridisirend nach den Gleichungen :

$$
\begin{aligned}
& \mathrm{SeCl}_{4}+2 \mathrm{SO}_{4} \mathrm{H}_{2}=\mathrm{SeOCH}_{2}+\mathrm{s}_{2} \mathrm{O}_{3} \mathrm{H}_{4}+2 \mathrm{ClH} ; \\
& \mathrm{SeOCH}_{2}+2 \mathrm{SO}_{4} \mathrm{H}_{2}=\mathrm{SeO}_{2}+\mathrm{8}_{2} \mathrm{O}_{7} \mathrm{H}_{2}+2 \mathrm{ClH} .
\end{aligned}
$$

Nach diesen zu schliefsen wäre Selenoxychlorid ein Zwischenproduct, kann aber nicht durch fractionirte Destillation isolirt werden, $d a$, wie ein kleiner Versuch zeigte, Selenoxychlorid und Schwefelsāore sich zwar in der Kälte mischen, ohne sich zu zersetzen, beim Erwärmen aber unter Abgabe von Salzsăure und Bildang von Pyroschwefelsäure sich umsetzen nach der letzten Gleichung (bei stärkerem Erhitzen sublimirt etwas $\mathrm{SeO}_{z}$ an die oberen Gefälswände).

\section{Pyrosulfurylchlorid und Selentetrachlorid.}

Auch Pyrosulfurylchlorid giebt sein Radical $\mathrm{SO}_{3}$ an Selentetrachlorid ab. Beide Stoffe wirkten, im Verhältnifs $1 \mathrm{Mol}$. auf 1 Mol. zusammengebracht, in der Kălte kaum auf einander ein, beim Erwärınen schäumte die Mischung so sehr, dafs ich noch eine 1 Mol. entsprechende Menge Pyrosulfurylchlorid hinzusetzen mufste. Das Selentetrachlorid löste sich in der Wärme vollständig, unter Abgabe eines constanten Stroms schwefliger Säure und Chlors. Beim Erkalten erstarrte die Masse nicht, weil das Pyrosulfurylchlorid noch von seiner Darstellung her etwas Schwefelsāureanhydrid gelōst enthielt, welches die Krystallisation hinderte. Sobald dasselbe abgeraucht war, bekam ich eben denselben Krystallkuchen wie aus Sulfurylhydroxylchlorid. Von bei der Umsetzung entstandenem Sulfurylchlorid konnte nichts entdeckt werden. Der Procefs geht einfach nach der Gleichung :

$$
\mathrm{S}_{8} \mathrm{O}_{6} \mathrm{Cl}_{2}+\mathrm{SeCl}_{4}=\mathrm{sO}_{3} \mathrm{BeCl}_{4}+\mathrm{SO}_{4}+\mathrm{Cl}_{4} \text {. }
$$


Das im Ueborschufs zugesetzte Pyrosulfurylchlorid diente wie früher Sulforylhydroxylchlorid (siehe oben) nur als Lossungsmittel.

So giebt es noch eine Anzahl Bildungsweisen des Schwefelselenoxytetrachlorids. Oxychlorid und Oxyd des Selens haben immer die Neigung, ihren Sauerstoff gegen Chlor der Schwefeloxychloride auszutauschen. Bs wirken z. B. :

\section{Bulfurylhydroxylchlorid und Selenoxychlorid}

schon in der Kälte heftig auf einander ein, unter BilJung von Selentetrachlorid und Pyroschwefelsäure und vielleicht (in Folge der Erwärmang bei der Reaction) von $\mathrm{SSeO}_{8} \mathrm{Cl}_{4}$ und Schwefelsāare; jedenfalls entstehen diess Producte, wenn man das Gemisch erwārmt. Ist genügend Sulfurylhydroxylchlorid vorhanden, so verlăuft, indem sich alles verflūssigt, der Procefs in folgenden zwei Phasen :

$$
\begin{gathered}
2 \mathrm{SO}_{2} \mathrm{Cl}(\mathrm{OH})+\mathrm{BaOCl}_{2}=\mathrm{BeCl}_{4}+\mathrm{s}_{2} \mathrm{O}_{7} \mathrm{H}_{2} ; \\
\mathrm{B}_{3} \mathrm{O}_{7} \mathrm{H}_{2}+\mathrm{BeCl}_{4}=\mathrm{BO}_{2} \mathrm{BeCl}_{4}+\mathrm{BO}_{4} \mathrm{H}_{2} .
\end{gathered}
$$

Das $\mathrm{SSeO}_{8} \mathrm{Cl}_{4}$ krystallisirt beim Erkalten aus der Lōsung ans.

Eulfurylhydroxylchlorid und Selenigsäureanhydrid

setzen sich nicht mit derselben Heftigkeit um; doch löst sich das Selenigsāureanhydrid beim Erwãrmen vollständig ohne erhebliche Abgabe von Gasen (Chlor und schweflige Sãure treten ais Zersetzungsproducto des Sulfurylhydroxylchlorids in geringer Menge auf). Beim Erkalten erhält man je nach der Menge des zugesetzten Sulfurylbydroxyichlorids einen mehr oder weniger licken Syrup, aus welchem man bei Destillation zuerst das überschūssige Hydroxylchlorid, später bei circa $180^{\circ}$ eine dicke, aus $\mathrm{SO}_{8}$ und $\mathrm{SSeO}_{3} \mathrm{Cl}_{4}$ bestehende hornartige Masse bekommt, welche mit etwas Schwefelsăure versetzt und so lange erhitzt, bis kein entweichendes $\mathrm{SO}_{8}$ mehr zu bemerken, in den bekannten strabligen Formen krystallisirt. 
Der Rũckstand von obiger Destillation bestand hauptsächlicb aus Scbwefelsāure, es ist somit die Reaction folgende :

$$
\begin{aligned}
& 2 \mathrm{BO}_{8} \mathrm{Cl}(\mathrm{OH})+\mathrm{BeO}_{2}=\mathrm{S}_{2} \mathrm{O}_{7} \mathrm{H}_{2}+\mathrm{B}_{6} \mathrm{OCl}_{2} \text {; } \\
& 2 \mathrm{8O}_{2} \mathrm{Cl}(\mathrm{OH})+\mathrm{BeOCl}_{2}=\mathrm{8}_{2} \mathrm{O}_{4} \mathrm{H}_{3}+\mathrm{BeCl}_{6} ; \\
& \mathrm{B}_{2} \mathrm{O}_{7} \mathrm{H}_{2}+\mathrm{8}_{0} \mathrm{Cl}_{4}=\mathrm{BO}_{2} \mathrm{SOCl}_{4}+\mathrm{SO}_{4} \mathrm{H}_{2} \text {. }
\end{aligned}
$$

Wie man sieht, entsteht nebenbei Pyroschwefelsäure, deren $\mathrm{SO}_{3}$ auch hier die Krystallisation des $\mathrm{SSeO}_{3} \mathrm{Cl}_{4}$ verhindert.

Analogie zurischen Selentetrachlorid, Schwefeltetrachlorid und Phosphorpentachlorid.

Während das Schwefelchlorid in seinem Verhalten sehr viel Aehnlichkeit hat mit Phosphorchlorid, ist die Analogie des Selenchlorids mit diesem schon viel geringer. Allerdings sehen wir auch bei ihm in der Hitze noch eine Abspaltung von Chlor und Bildung eines niederen Chlorids, es läfst sich auch noch ein Oxychlorid von ihm ableiten, aber der Austausch seines Chlors gegen Sauerstoff geht schon viel schwieriger als bei den Chloriden des Phosphors und Schwefels. Diese zersetzen sich 2. B. mit Schwefelsãureanhydrid nach den Gleichungen :

$$
\begin{aligned}
& \mathrm{PCl}_{6}+\mathrm{BO}_{3}=\mathrm{POCl}_{8}+\mathrm{SO}_{3}+\mathrm{Cl}_{2} ; \\
& \mathrm{SCl}_{4}+\mathrm{SO}_{3}=\mathrm{SOCl}_{3}+\mathrm{SO}_{2}+\mathrm{Cl}_{2} .
\end{aligned}
$$

Selentetrachlorid tauscht 2 Aeq. Chlor gegen 2 Aeq. Sauerstoff nur sehr selten aus, so wenn diese 2 Aeq. Sauerstoff mit weiteren zwei an ein Atom Selen gebunden sind, wo also ein einfacher Ausgleich stattindet :

$$
\mathrm{BeCl}_{4}+\mathrm{SeO}_{3}=\mathrm{SeOCl}_{4}+\mathrm{SeOCl}_{3}
$$

oder wenn das Chlor im Stande ist, mit Wasserstoff verbunden als Saizsăure zu entweichen, wenn sich also Selentetrachlorid mit Wasser oder dessen Bestanơtheilen unsetzen kann wic bei Anhydrisirung, wobei sogar alle $4 \mathrm{Aeq}$. Chlor gegen $4 \mathrm{Aeq}$. Sauerstoff ausgetauscht werden hönnen, z. B. :

$$
\begin{aligned}
& \mathrm{SeCl}_{4}+2 \mathrm{SO}_{4} \mathrm{H}_{2}=80 O \mathrm{SO}_{3}+\mathrm{S}_{2} \mathrm{O}_{7} \mathrm{H}_{2}+2 \mathrm{ClH} ; \\
& \mathrm{SoOCl}_{2}+2 \mathrm{BO}_{4} \mathrm{H}_{2}=\mathrm{SoO}_{2}+\mathrm{S}_{2} \mathrm{O}_{4} \mathrm{H}_{2}+2 \mathrm{CHA} .
\end{aligned}
$$


Unter Umstānden giebt Selentetrachlorid auch nur ein Atom Chlor ab und bindet dafür ein einwerthiges Radicel :

$$
\mathrm{SoCl}_{4}+\mathrm{SO}_{2}=\mathrm{Cl}_{3} \mathrm{Se}-\mathrm{O}-\mathrm{8O}_{2} \mathrm{Cl} \text {. }
$$

Phosphorpentachlorid zeigt eine derartige Umsetzung gar nicht, Schwefeltetrachlorid nur im günstigsten Fall (siehe oben).

Noch schärfer zeigt sich der Unterschied im Verhalten der Chloride bei Einwirkang auf organische hydroxylhaltige Substanzen.

Phosphorpentachlorid zersetzt sich leicht mit Säuren und giebt Phosphoroxychlorid und Salzsäure, Schwefeltetrachlorid giebt, wie Carius*) zeigte, analoge Producte nach den Gleichungen :

$$
\begin{aligned}
& \mathrm{PCl}_{6}+\mathrm{CH}_{8} \mathrm{COOH}=\mathrm{POCl}_{8}+\mathrm{CH}_{8} \mathrm{COCl}+\mathrm{CH} ; \\
& \mathrm{gCl}_{6}+\mathrm{C}_{6} \mathrm{H}_{6} \mathrm{COOH}=\mathrm{SOCl}_{2}+\mathrm{C}_{6} \mathrm{H}_{5} \mathrm{COCl}+\mathrm{ClH} .
\end{aligned}
$$

Selentetrachlorid wirkt auf Essigsãure (krystallisirharen Bisessig) bei gewöhnlicher Temperatur fast gar nicbt ein ; erwärmt man, so löst es sich unter Abgabe von Salzsture und allmäliger Gelbfärbung. Bis sich alles gelōst hat, ist die Masse schon dunkelbraunroth von gebildetem Selenchlorür, läfst man nun erkalten, so krystallisirt das gelöste aber noch unzersetzte Selentetrachlorid in der schon oben beschriebenen Form aus, ebenso aus den Destillaten, wenn man jetzt die Masse, welche als dunkelrothe Flüssigkeit übergeht, abdestillirt. Erhitzt man am Rũckflufskühler weiter, so wird die Flüssigkeit unter weiterer Salzsăureabgabe wieder hell rosenroth, dabei scheidet sich ein Selenregulus am Boden ab. Ganz entfärbt sich die Flüssigkeit selbst bei stundenlangen Kochen nicht. Destillirt man nun ab, so bekommt man rothe, zwischen 110 und $150^{\circ}$ übergehende Destillate, welche aus überschüssiger Essigsäure und Chlorsulustitutionsproducten der Essigsäure resp. ihres Anhydrids zu bestehen, aber durch unzersetztes

*) Digre Annalen 196, 292. 
Selenchlorür in Siedepunkt alterirt scheinen. Der Destillationsrückstand besteht aus Selen und Selenigsãureanhydrid, welche sich in stärkerer Hitze sublimiren lassen. Acetylchlorid, dessen Entstehung man nach Analogie mit Phosphorpentachlorid und Schwefeltetrachlorid hälte erwarten können, kann sich gar nicht gebildet haben, wie ein direct angestellter Versuch mir bewies; denn jeder in Acetylchlorid fallende Tropfen Selenoxyehlorid setzte sich, wie früher mit Sulfurylhydroxylchlorid, rasch und heftig damit um in Essigsāureanhydrid und Selentetrachiorid, welches letzlere sich ausschied und beim Erhitzen unter denselben Erscheinungen, wie früher in Essigsāure, löste. Feste Producte bekam ich nicht. Es scheinen bei der Einwirkung von Selentetrachlorid auf Essigsăure zweieriei Processe neben einander herzugehen, Chlorirung und Verwandlung der Săure in Anhydrid *), letztere nach der Gleichang : $2 \mathrm{C}_{3} \mathrm{~B}_{4} \mathrm{O}_{3}+\mathrm{SeCl}_{4}=\mathrm{C}_{4} \mathrm{H}_{8} \mathrm{O}_{3}+86 \mathrm{OCl}_{3}+2 \mathrm{ClH}$.

Ist kein $\mathrm{SeCl}_{4}$ mehr vorhanden, so zersetzt sich $\mathrm{SeOCl}_{8}$ weiter nach der Gleichung :

$$
2 \mathrm{C}_{3} \mathrm{~B}_{6} \mathrm{O}_{3}+\mathrm{S}_{6} \mathrm{OCl}_{2}=\mathrm{C}_{4} \mathrm{~B}_{6} \mathrm{O}_{8}+\mathrm{B}_{6} \mathrm{O}_{4}+2 \mathrm{ClH} \text {. }
$$

Nebenbei aber giebt Selentetrachlorid unter Bildung von Selen-

*) Phosphortriahlorid wirkt, wie Buckney und Thomsen beim Einwirkenlassen auf Trichloressigskure fanden, anch schwach wasserentziebend (Berichte der deutechen chomischen Gesellschaft 10, 698). Dafs die orste Einwirkung ron Phospherpentachloria in einer Wasserentziehung bestehe und erst im zweiten Stadium der Resction ein chlorhaltiger Körpor erzengt werde, glaubten schon Gerhardt und Cbiosza (Ladenbarg, Entwickelungsgeschiohte der Chemis 1869, 227 ; Compt. rend. 8s, 1050) aus ihren Versuohen nachweisen su können, dafs aber Phopphurpentachlorid in der That oinfach anhydridisirend wirken kann, hat Wfllisisn s (Jahresber. für Chemie a. s. w. f. 1869, 207 ; Journal ftir praktische Chemio 100, 124) gexeigt, welcher zu viel Bchwofelskuro, wenig Phosphorchlorid brachte und anstats Sulfurylbydroxylohlorid nur Schwefelelureanhydrid resp. Pyronohwefolsture bekam. 
chlorür und sogar von Selen sein Chlor $\mathrm{b}$, welches wie freies Chlor wirkt, d. h. die Wasserstoffatome im $\mathrm{CH}_{8}$ substituirt und so die Essigsäure in die gechlorten Essigsäuren überführt.

Sulfurylhydroxylchlorid und Titantetrachlorid.

Als ich $2814 \mathrm{Grm}$. Titanchlorid (entsprechend $1 \mathrm{Mol}$.) tropfenweise $8,1 \mathrm{Grm}$. Sulfurylhydroxylchlorid (entsprechend 1 Mol.) fügte, erzeugte jeder Tropfen sogleich unter schwacher Salzsãureabgabe eine zu Boden fallende gelbe Haut; nachdem alles Sulfurylhydroxylchlorid zugegeben war, schien das Product nahezu fest und mit wenig Flüssigkeit durchtrānkt. Beim Erwārmen in Wasserbad aber bläbte sich dio Masse auf und entliefs reichlich Salzsäuredämpfe, indem es zum trockenen amorphen Pulver wurde, welches unter Abgabe von etwas Chlor und schwefliger Sãure mit Wasser schwach zischi, mit wenig zur weifsen Masse werdend, mit mehr sich zur klaren, hellen Flüssigkeit lōsend. Bs raucht an feuchter Luft und wird za einer weifsen feuchten pulverigen Masse, weiche nun in Wasser sich nicht mehr vollstāndig lōst. Ein Trocknen des Pulvers vor der Analyse zwischen Thonplatten schien unnöthig. Zur Analyse selbst brachte ich in zwei getrennten Portionen die Substanz in mit Glasstōpsel versehene tarirte Wägeröhrchen; letztere öffnete ich nach Einschmelzen in eine mit Ammoniaklösung halbgefüllte Röhre durch Abschütteln des Stöpsels resp. Ausschlagen des dūnnen Bodens. Die Reaction mit Ammoniak war eine sehr heftige. Nach Filtration von der ausgefâllten Titansäure wurde mit salpetersaurem Ammoniak ausgewaschen, im Filtrat das eine Mal nach Oxydation mit Chlor, um etwa vorhandene schweflige Sãure in Schwefelsāure überzuführen, die Schwefelsäure, das andere Mal das Chlor und dann die Schwefelsäure gefällt.

I. 0,9851 Grm. Substanz lieferten 0,3052 $\mathrm{TiO}_{2}$ and 0,8158 $\mathrm{BaSO}_{4}$.

II. $0,5890, " \quad$ " $\quad, 1831 \mathrm{TiO}_{2}, 0,4765 \mathrm{BaBO}$, und 


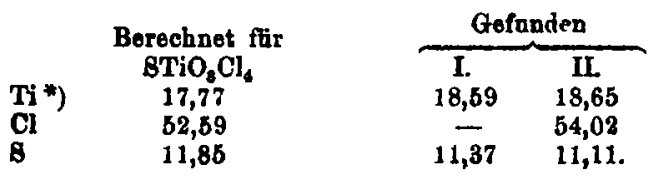

Ich habe somit hier die dem Schwefeloxytetrachlorid analoge Verbindung Schwefeltitanoxytetrachlorid :

$$
\mathrm{ClSO}_{\mathbf{2}}-\mathrm{O}-\mathrm{TiCl}_{3} \text {. }
$$

Beim Erhitzen auf höhere Temperatur zersetzt sich der Körper gröfstentheils, unter Zurücklassung chlorfreier Titansäure und Ausstofsung eines weirsen, in abgekūhlter Yorlage sich theilweise zu Tröpfchen verdichtenden Rauchs; Chlor und etwas schweflige Săure entweichen; ein kleiner Theil der Verbindung scheint unverändert als gelbes Häutchen zu sublimiren. Bei gewöhnlicher Temperatur zersetzt sich das Pulver nicht von selbst, es verändert sein Aussehen nicht wăhrend monatelangen Stehens am zerstreuten Tages- und Sonnenlicht.

Nach den Versuchen von $W$ e ber ${ }^{* *}$, welcher die Doppelverbindungen $\mathrm{SnCl}_{4}, 2 \mathrm{SeOCl}_{2} ; \mathrm{TiCl}_{4}, 2 \mathrm{SeOCl}_{2} ; \mathrm{SbCl}_{5}$, $2 \mathrm{SeOCl}_{2}$, deren Constitution wohl folgendes Schema hat :

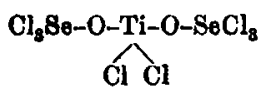

darstellte durch Zusammenbringen der entsprechenden Chloride mit Selenoxychlorid, könnte man erwarten, dafs sich Antimonpentachlorid und Zinntetrachlorid auch dem Sulfurylhydroxylchlorid gegenüber ähnlich dem Titantetrachlorid verhielten; diefs ist aber nicht der Fall.

Sulfurylhydroxylchlorid und Antimonpentachlorid mischten sich im Fractionskolben, im Verhăltnifs 1 Mol. auf 1 Mol. zusammengebracht, vollstāndig zur grūnlichgelben Flüssigkeit. Bei schwachem Brwärmen schon färbte sich die Masse helibraun, unter Abgabe von Strōmen Chlors und

*) Atomgewicht des Titans gleich 48 angenommen.

**) Pogg. Ann. 105, 825. 
schwefliger Säure neben Salzsäure. Nach einiger Zeit schied sich ein fester Körper aus und bei Wegnahme der Flamme erstarrte 3/. der Flüssigkeit zur gelhlichweifsen Krystallmasse. Bei nochmaligem stärkerem Erwärmen löste sich wieder ein Theil, die Entwickelung von Chlor und schwefliger Sãure begann abermals, bei circa $160^{\circ}$ destillirte etwas Flüssigkeit. Als Rückstand blieb eine weifse krystallinische Masse, welche zwischen Thonplatten vollends getrocknet werden konnte. Das trockene Pulver raucht nicht an der Luf, zerfliefst aber aufserordentlich rasch und giebt mit mehr Wasser ohne heftige 7ersetzung oder Auftreten von Gasen eine starke weifse Ausscheidung von Antimonsäure. Zur Chlor- und Schwefelbestimmung wurde ein Wägeröhrchen mit der abgewogenen Substanz unter verdünnter Weinsăure geöfinet. Ich erhielt folgende Zahlen :

\begin{tabular}{|c|c|c|}
\hline & $\begin{array}{l}\text { Berechnet für } \\
\mathrm{SO}_{\text {s ShCl }}\end{array}$ & Gofunden \\
\hline $\mathrm{Cl}$ & $4 \epsilon, 8$ & 39,05 \\
\hline $\mathbf{s}$ & 8,4 & b,34. \\
\hline
\end{tabular}

Die massenhaft auftretenden Gase Chlor und schweflige Sãure, welche nicht allein herrūhren können von der Zersetzung des Sulfurylhydroxylchlorids und die Resultute der Analyse zeigen, dafs man auf diesen Wege nicht zu der Verbindung :

$$
\mathrm{CISO}_{4}-\mathrm{O}-\mathrm{BbCl}_{4} \text {, }
$$

sondern zu Zersetzungsproducten, einem Gemisch von vielleicht allen moglichen Chlor-, Chlor- und Sauerstoff- und schwefelsuuren 0xydverbindungen *) des Antimons gelangt. Durch qualitative Probe fand ich Antimon als Antimonsäure and Antimonoxyd vorhanden.

Im Einschmelzrohr iñi Verhältnirs 1 Mol. auf 1 Mol. ge-

*) Williama bat moi Oxychlorido der höheren Qxydationestufo dos Autimons dergentellt and isolirt: $\mathrm{Sb}_{3} \mathrm{Cl}_{1}, \mathrm{O}$ und $\mathrm{Sb}_{3} \mathrm{Cl}_{9} \mathrm{O}_{2}$ (Jahresber. für Chemio u. o. w. f. 1871,329 ). 
mischt, wirken Antimonchlorid und Sulfurylhydroxylchlorid bei mehrstūndigem Erhitzen im $W$ asserbad und monatelangem Stehen im Sonnenlicht nicht auf einander ein. Ist das Sulfurylhydroxylchlorid schon öfter mit feuchter Lnft in Berührung gekommen, so entsteht bald nach dem Zusammenbringen mit Antimonchlorid ein Niederschlag, welcher aber lediglich ein Product der Einwirkung gebildeter Schwefelsäure auf Antimonchlorid ist. Letzteres nämlich und Schwefelsäure mischen sich beim Zusammenbringen anfänglich gar nicht, allmälig geben sie unter Salzsäureentwickelung eine Emulsion, welche nach wochenlangen Stehen ein faseriges seideglänzendes Aussehen annimint; doch geht die vollständige Zersetzung so langsam, dafs vorerst eine Analyse unmöglich erscheint.

Sulfurylhydroxylchlorid und Antimontrichlorid.

Antimontrichlorid lōst sich in Sulfurylhydroxylchlorid bei gewöhnlicher Temperatur nur in geringer Menge; erhitzt man das Gemisch im Einschmelzrohr, so schmilzt das Antimonchlorür und sammelt sich als untere Flüssigkeitsschicht, bej höherer Temperatur mischen sich auch die beiden Flüssigkeiten, beim Erkalten krystallisirt aber das Antimonchlorür unverändert wieder aus.

\section{Sulfurylhydroxylchlorid und Zinntetrachlorid}

in gleicher Weise im Einschmelzrohr zusanmengebracht mischten sich nur theilweise und zwar je nach der Temperatur in verschiedenem Verbältnifs. Erhitzen während mehrerer Stunden auf $170^{\circ}$ und längeres Stehenlassen im Sonnenlicht ånderte Nichts an der Sache.

Rose *) hatte aus $\mathrm{SnCl}_{4}$ und $\mathrm{SO}_{3}$ eine feste Verbindung $4 \mathrm{SnOC}_{2}, 5 \mathrm{~S}_{2} \mathrm{O}_{6} \mathrm{Cl}_{2}$ erhaiten.

*) Gmelin, Handb. d. Chem. 6. Auf., 8, 185; Pogg. Ann. 44, 320. 
Sulfurylhydroxylchlorid und Biliciumtetrachlorid.

Siliciumchlorid, dargestellt durch Glähen von Kohle und Kieselguhr im Chlorstrom und einmalige Destillation des Rohproducts verhielt sich gegen Sulfurylhydroxylchlorid ganz wie Zinnchlorid.

Gustayson *) bekam aus $\mathrm{SiCl}_{4}$ and $\mathrm{SO}_{8}$ Pyrosulfurylchlorid; es scheinen somit derartige Chloride (auch $\mathrm{CCl}_{4}$ ) **) nur schwierig mit $\mathrm{SO}_{8}$ in Reaction zu treten, wenn aber einmal Zersetzung eintritt, so schreitet sie mit Uebergehung der intermediären Doppelverbindung weiter.

Einwirkung von Bromwasserstoff auf Schwofelsäureanhydrid.

Zu den dem Sulfurylhydroxylchlorid und Schwefeloxytetrachlorid entsprechenden Bromverbindungen suchte ich auf verschiedenen Wegen zu gelangen, olne andere als negative Resultate zu bekommen.

Ueber eine schwefelsaure Bromwasserstoffsăure" existirt nur eine kleine Notiz von A i me ${ }^{* * *}$, welcher Bromwasserstoff auf Schwefelsäureanhydrid einwirken liefs. Auch ich versuchte diese beiden mit einander zu verbinden. Ich destillirte in eine mit eingeriebenen, durch Glashahnen verschliefsbaren Rōhren versehene $\mathrm{W}_{0}$ u If e'sche Flasche $\mathrm{SO}_{3}$ und leitete dann trockene, aus Phosphorbromür und Wasser dargestellte Bromwasserstoffsāure durch das auf $0^{\circ}$ abgekühlte Gefáfs. Bald konnte man sehen, wie sich das Schwefelsäureanhydrid roth färbte und zerflors; die entweichenden Gase bestanden aus überschūssiger Bromwasserstoffsăure, Brom und schwefliger Sāure. Nach mehrstūndigem Hindurchleiten von Bromwasserstoff war nur noch wenig Flüssigkeit in der Flasche

*) Compt. rend. $26,92$.

**) Soh titzonbergor, Zeitsohrift fir Chomio 1869, 631 .

w**) Gmolin, Bandb. d. Chem. 6. Aufl, 1, 2, 8. 338; Joum. Pharm. 81, 88. 
bestehend auc zwoi Schichten, 1) einer oberen hellen, der Hauptsache nach concentrirte Schwefelsäure mit etwas gelöstem Brom und schwefliger Săure, und 2) einer unteren dunkelen, etwas nach Bromschwefel riechenden, im Wesentlichen aus Brom bestehend. Der Procéfs verlâuft also nicht nach der Gleichung :

sondern in folgender Weise :

$$
\mathrm{SO}_{2}+\mathrm{BrH}_{\mathrm{r}}=\mathrm{BO}_{2} \mathrm{Br}(\mathrm{OH})
$$

$$
\begin{aligned}
& \mathrm{BO}_{2}+3 \mathrm{BrH}=\mathrm{BO}_{2}+\mathrm{Br}_{2}+\mathrm{E}_{2} \mathrm{O} \text {; } \\
& \mathrm{SO}_{3}+\mathrm{H}_{4} \mathrm{O}=\mathrm{SO}_{4} \mathrm{H}_{\text {. }}
\end{aligned}
$$

Wabrscheinlich wird elwas schweflige Sãure noch weiter reducirt nach der Gleichung :

$$
\left.\mathrm{SO}_{3}+4 \mathrm{BrH}=\mathrm{BBr}_{4}+2 \mathrm{~B}_{2} \mathrm{O}^{*}\right) \text {. }
$$

Einwoirkung von Phosphorpentabromid auf Bchwoofalsiure.

Ein zweiter Versuch, Sulfurylhydroxylbromid zu bekommen durch Einwirkenlassen von Phosphorbromid auf Schwefotsãure (analog der Darstollung von $\mathrm{SO}_{2} \mathrm{Cl}(\mathrm{OH})$ aus $\mathrm{PCl}_{5}$ und $\mathrm{SO}_{4} \mathrm{H}_{2}$ ), fübrte zu einem ăhnlichen negativen Resultat. Ich trug in eine Retorte mit Rückflufskühler und Chlorcalciumverschlufs zu $300 \mathrm{Grm}$. Schwefelsăure vom spec. Gewicht 1,85 bei $20^{\circ} 278 \mathrm{Grm}$. Phosphorbromid portionenweise ein. Letzteres sank in der kalten Schwefelsãure zu Boden und löste sich erst bei gुelindem Erwärmen unter Abgabe von Bromwasserstoff, Brom und schwefliger Saure und Rothfartbung der Flüssigkeit. Nachdem alles Phosphorbromid eingetragen war wurde abdestillirt, es ging ziemlich constant bei $67^{\circ}$ eine rothe Flüssigkeit, welche im Wesentlichen aus Brom bestand und etwas nach Bromschwefel roch, über; als Gase entwichen Bromwasserstoffsāure, schweflige Sãure and etwas Brom. Zuletzat

*) Trookene Jodwasserstoffishure und Chlorwaseonto fishure zervetzon sioh mit trockener schwefliger Bzure zu Wasser, Bchwofel und Jod resp. Chlor; bei Gegenwart ron Wasser findet keine Zorsotzong statt (D u mas, Traité d. Chim. 1, 146; Gmolin, Handb. d. Chem. 6. Anf., I, 2, S. 184). 
wurde der Retorteninhalt hell und dickflussig und bestand schlierslich aus einem Gemisch von Phosphorsäure und Schwefelsăure.

Einwirkung von Schwefeltetrabromid auf Schwefelsäureanhydrid.

In gleich negativem Sinne verlief der Versuch, Sthwefeloxytetrabromid herzustellen.

Rose *) thut eines mifslungenen Versuchs Erwăhnung, weichen er ausgeführt, um durch Vereinigung einer Lösung von Schwefel in Brom mit Schwefelsāureanhydrid zu einer Doppelverbindung zu gelangen. Er bemerkte dabei kein Auftreten von schwefliger Sãure.

Ich brachte bei einer Temperaiur von $+6^{\circ} \mathrm{zu}$ verschiedenen Malen eine Lösung von 1 Alomgew. Schwefel in $4 \mathrm{Atom-}$ gew. Brom (also Schwefeltetrabromid) zu Schwefelsäureanhydrid im Verhăltnifs 1 Mol. auf 1 Mol. und konate beobachten, wie allmälig das Schwefelsäureanhydrid zerflofs und durch ein dem Gefüfs aufgesetztes Chlorcalciumrohr ein bräunliches Gas, bestehend aus schwefliger Săure und Brom (Sauerstoff nicht vorhanden), in sehr constantem Strom entwich, bis nach einigen Tagen alles Schwefelsäureanhydrid zu einer aus zwei Schichten bestehenden Flüssigkeit gelöst war; die obere hellere war ein Gemisch aus Schwefe!säureanhydrid, schwefliger Säure und Brom, die untere dunkle bestand aus Brom und unzersetztem Bromschwefel. Die Rtaction erfolgt somit nach der Gleichung :

$$
2 \mathrm{BO}_{3}+\mathrm{BBr}_{6}=3 \mathrm{SO}_{4}+2 \mathrm{Er}_{6} .
$$

Schliefslich sage ich meinem hochverehrten Lehrer, Herrn Professor Dr. Loth. Meyer, auf dessen Anregung und unter dessen Leitung vorstehende Untersuchungen unternommen wurden, herzlichen Dank für seine werthvollen Ratbschläge und die stets wohlwollende Unterstützung bei Ausführung der Arbeiten.

*) Gmelin, Handb. d. Ch. 6. Auf., 1, 2, S. 338; Pogg. Ann. 44, 327. 